\section{Carotid Endarterectomy: Which Technique Prevails?}

\section{Abstract}

For decades, carotid endarterectomy has been the golden standard for the management of severe carotid artery stenosis. However, several techniques have been proposed till date, and there has been a long-lasting debate concerning the proper method. Several research studies have been conducted not only evaluating the role of patch suturing versus that of primary carotid closure, but also comparing the advantages and disadvantages of different patch materials. Both synthetic and venous patches have been utilized, each with specific features and effects on postoperative outcomes. This editorial aims to summarize basic characteristics of each technique, current guidelines recommendations and pooled research data on this subject, and produce conclusions for everyday clinical practice.

Keywords: Carotid endarterectomy; Eversion; Conventional; Patch

\section{George Galyfos, Georgios Geropapas, Stavros Kerasidis and Georgios Kastrisios}

\author{
Department of Vascular Surgery, KAT \\ General Hospital, Athens, Greece
}

\section{Corresponding author: George Galyfos}

\section{georgegalyfos@hotmail.com}

2 Nikis Street, Kifisia, 14561, Athens, Greece.

\section{Tel: $+30-213-2086243$}

Fax: +30-213-2086445

Citation: Galyfos G, Geropapas G, Kerasidis $\mathrm{S}$, et al. Carotid Endarterectomy: Which Technique Prevails? Journal of Vascular \& Endo Surgery. 2016, 1:1.

data indicate so far that $10 \%$ - $20 \%$ of all cerebra ischemia cases are due to atherosclerotic lesions within the extracranial carotid circulation [1]. Cerebrovascular ischemic events remain still the $3^{\text {rd }}$ cause of death worldwide and a primary cause for neurologic dysfunction in adults as well [1]. Therefore, they are associated with significant morbidity and mortality. Major clinical trials have shown that carotid endarterectomy (CEA) is beneficial both for primary and secondary prevention of such acute cerebrovascular events in patients with severe carotid artery stenosis $[2,3]$. However, for decades, there has been a debate regarding the proper surgical technique.

For years, the traditional method has been the conventional CEA, either through a primary suturing technique or a patching technique. Furthermore, patches could be synthetic (either Dacron or PTFE material) or venous in origin. However, another alternative that has been introduced almost at the same time with the conventional procedure was the eversion CEA. It is clear that each one of the aforementioned techniques has specific advantages and disadvantages. Primary suturing is associated with lower duration of procedure as well as clamping time, thus reducing possible complications linked to periprocedural cerebral ischemia. However, patching increases the postoperative diameter of the vessel and minimizes the effect of neointimal hyperplasia and scarring postoperatively, thus decreasing the risk for potential restenosis. Moreover, standard patching has been shown that it reduces the perioperative stroke and death risk [4]. Regarding the patch selection, synthetic patches are readily available all the time and are more resistant than venous patches. However, venous patches show excellent handling, are less prone to thrombosis formation or restenosis due to their endothelial lining on the luminal surface, and hence, the risk for pseudoaneurysms formation or patch infection is minimized [5]. Finally, the eversion technique removes the atherosclerotic plaque after transection of the internal carotid artery at its origin. This technique offers again reduced time of procedure and clamping, it avoids suturing distally on the internal carotid artery, avoids the potential complications of suturing a foreign material, and it seems that is associated with better hemodynamics $[6,7]$. But what do the guidelines recommend and what do the pooled data reveal so far?

According to the latest ESVS Guidelines, patch angioplasty (with either venous or synthetic patch) may reduce the risk for carotid restenosis and ischemic stroke, compared to primary closure, and therefore it is recommended [1]. Likewise the American Guidelines conclude that patch angioplasty can be beneficial for CEA closure (Level of Evidence: B) [8]. Currently, in other advanced countries such as Germany, the official Guidelines recommend the standard suturing of a patch as it is associated with lower perioperative stroke / death rates as well as postoperative restenosis rates. However, they indicate that the choice between conventional and eversion technique should be made based on the experience of the responsible physician [9]. Finally, in the United Kingdom (UK), 
only one third of the physicians select to suture a patch during CEA, while almost $70 \%$ use a synthetic patch of various materials [10]. Hence, less than $10 \%$ of physicians in UK select to proceed with an eversion technique [10].

Regarding the available pooled data so far, the first major metaanalysis comparing eversion with conventional CEA was that by Cao et al. in 2002 [11]. As only five trials and 2,465 patients were included, the authors produced no definite results concluding that eversion technique may be associated with lower risk of occlusion and restenosis, although a larger number of cases would be necessary to support this further. Therefore, the selection of the technique was suggested to be based on the preference of each physician. Two years later, another review by Bond et al., included 7 trials comparing primary suturing with patch technique, and 8 trials comparing different types of patches [12]. Although this review concluded definitely in favour of the patch suturing versus primary closure, no robust conclusion could be drawn concerning the type of material. More recent pooled data by Rerkasem and Rothwell seems to favour standard patching although it is underlined that all included trials were small and methodological errors have been observed [13].

However, the two most recent meta-analyses are the largest in size to date and seem to shed some light concerning the debate on proper technique and patch type. Antonopoulos et al. included more than 16,000 patients and compared the eversion against the conventional CEA [14]. Seven randomized and fourteen nonrandomized trials were analyzed and the authors concluded that eversion CEA seems to be superior to conventional CEA regarding short - term and long - term outcomes, without being affected by potential modifying factors. Additionally, Ren et al. [15] collected data from thirteen randomized trials evaluating the effect of different patch types on major outcomes. Almost 2,000 patients were included and only haemostasis time was found to be longer with PTFE patches compared to venous or Dacron patches. However, the overall perioperative and long-term mortality rate, stroke rate, restenosis, and operative time were similar when using venous patch versus synthetic patch material or Dacron patch versus PTFE patch material during CEA, according to the authors [15].

Finally, novel techniques have been developed in order to improve the aforementioned results of each method. Modified eversion techniques have been proposed by some authors yielding satisfying early and late results [16]. Furthermore, microsurgical non-patch techniques utilizing a surgical microscope have shown low midterm restenosis rates and comparable 30 day mortality / morbidity to other patching techniques [17].

In conclusion, data so far indicate that patch CEA should be preferred than primary suturing overall, although pooled results on the performance of different patch materials are still inconclusive. Concerning eversion technique, pooled data are in favour although larger series are needed to produce safer results. Therefore, international Guidelines recommend that the selection of the technique should be mainly based on surgeon's preference and experience. 


\section{References}

1 Liapis CD, Bell PR, Mikhailidis D, Sivenius J, Nicolaides A, et al. (2009) ESVS Guidelines Collaborators. Eur J Vasc Endovasc Surg 37: 1-19.

2 Halliday A, Mansfield A, Marro J, Peto C, Peto R, et al. (2004) MRC Asymptomatic Carotid Surgery Trial (ACST) Collaborative Group. Prevention of disabling and fatal strokes by successful carotid endarterectomy in patients without recent neurological symptoms: randomised controlled trial Lancet 363: 1491-1502.

3 North American Symptomatic Carotid Endarterectomy Trial Collaborators (1991) Beneficial effect of carotid endarterectomy in symptomatic patients with high-grade carotid stenosis. N Engl J Med 325: 445-453.

4 Muto A, Nishibe T, Dardik H, Dardik A (2009) Patches for carotid artery endarterectomy: current materials and prospects. J Vasc Surg 50: 206-213.

5 Mannheim D, Weller B, Vahadim E, Karmeli R (2005) Carotid endarterectomy with a polyurethane patch versus primary closure: a prospective randomized study. J Vasc Surg 41: 403-407.

6 Kamenskiy AV, Pipinos II, Dzenis YA, Gupta PK, Jaffar Kazmi SA, et al. (2013) A mathematical evaluation of hemodynamic parameters after carotid eversion and conventional patch angioplasty. Am J Physiol Heart Circ Physiol 305: 716-724.

7 Demirel S, Macek L, Bruijnen H, Hakimi M, Böckler D, et al. (2012) Eversion carotid endarterectomy is associated with decreased baroreceptor sensitivity compared to the conventional technique. Eur J Vasc Endovasc Surg 44: 1-8.

8 Brott TG, Halperin JL, Abbara S, Bacharach JM, Barr JD, et al. (2011) American College of Cardiology Foundation / American Heart Association Task Force on Practice Guidelines; American Stroke Association; American Association of Neuroscience Nurses; American Association of Neurological Surgeons; American College of Radiology; American Society of Neuroradiology; Congress of Neurological Surgeons; Society of Atherosclerosis Imaging and Prevention; Society for Cardiovascular Angiography and Interventions; Society of Interventional Radiology; Society of Neuro Interventional Surgery; Society for Vascular Medicine; Society for Vascular Surgery; American Academy of Neurology and Society of Cardiovascular Computed Tomography. 2011 ASA / ACCF / AHA /
AANN / AANS / ACR / ASNR / CNS / SAIP / SCAI / SIR / SNIS / SVM / SVS guideline on the management of patients with extracranial carotid and vertebral artery disease: executive summary. Stroke 42 : 420-463.

9 Eckstein HH, Kühnl A, Dörfler A, Kopp IB, Lawall H, et al. (2013) Multidisciplinary German-Austrian guideline based on evidence and consensus. The diagnosis, treatment and follow-up of extracranial carotid stenosis. Dtsch Arztebl Int 110: 468-476.

10 Rudarakanchana N, Halliday AW, Kamugasha D, Grant R, Waton S, et al. (2012) Carotid Endarterectomy Steering Group; Clinical Effectiveness Evaluation Unit, Royal College of Physicians; Vascular Society of Great Britain Ireland Current practice of carotid endarterectomy in the UK. Br J Surg 99: 209-216.

11 Cao P, De Rango P, Zannetti S (2002) Eversion vs. conventional carotid endarterectomy: a systematic review. Eur J Vasc Endovasc Surg 23: 195-201.

12 Bond R, Rerkasem K, Naylor AR, Aburahma AF, Rothwell PM (2004) Systematic review of randomized controlled trials of patch angioplasty versus primary closure and different types of patch materials during carotid endarterectomy. J Vasc Surg 40: 1126-1135.

13 Rerkasem K, Rothwell PM (2011) Systematic review of randomized controlled trials of patch angioplasty versus primary closure and different types of patch materials during carotid endarterectomy. Asian J Surg 34: 32-40.

14 Antonopoulos CN, Kakisis JD, Sergentanis TN, Liapis CD (2011) Eversion versus conventional carotid endarterectomy: a metaanalysis of randomised and non-randomised studies. Eur J Vasc Endovasc Surg 42: 751-765.

15 Ren S, Li X, Wen J, Zhang W, Liu P (2013) Systematic review of randomized controlled trials of different types of patch materials during carotid endarterectomy. PLoS One 8: 55050.

16 Abud B, Talay S, Ünal CS, Kubat E, Karaarslan K, et al. (2015) Comparison of the Modified Eversion Carotid Endarterectomy Technique with the Conventional Carotid Endarterectomy Technique: Early Results. World Journal of Cardiovascular Diseases 5: 87-94.

17 Reinert M, Mono ML, Kuhlen D, Mariani L, Barth A, et al. (2012) Restenosis after microsurgical non-patch carotid endarterectomy in 586 patients. Acta Neurochir (Wien) 154: 423-431. 